\title{
An AC-Rich Bean Element Serves as an Ethylene-Responsive Element in Arabidopsis
}

\author{
Chunying Wang ${ }^{\dagger}$, Tingting Lin ${ }^{\dagger}$, Mengqi Wang and Xiaoting Qi * \\ College of Life Science, Capital Normal University, Beijing 100048, China; 2190801018@cnu.edu.cn (C.W.); \\ zoetingy@gmail.com (T.L.); mitchkeyblues@gmail.com (M.W.) \\ * Correspondence: qixiaoting@cnu.edu.cn \\ + These authors contribute equally.
}

Received: 17 July 2020; Accepted: 11 August 2020; Published: 14 August 2020

\begin{abstract}
Ethylene-responsive elements (EREs), such as the GCC box, are critical for ethylene-regulated transcription in plants. Our previous work identified a 19-bp AC-rich element (ACE) in the promoter of bean (Phaseolus vulgaris) metal response element-binding transcription factor 1 (PvMTF-1). Ethylene response factor 15 (PvERF15) directly binds ACE to enhance PvMTF-1 expression. As a novel ERF-binding element, ACE exhibits a significant difference from the GCC box. Here, we demonstrated that ACE serves as an ERE in Arabidopsis. It conferred the minimal promoter to respond to the ethylene stress and inhibition of ethylene. Moreover, the cis-acting element ACE could specifically bind the nuclear proteins in vitro. We further revealed that the first 9-bp sequence of $\mathrm{ACE}\left(\mathrm{ACE}_{\mathrm{core}}\right)$ is importantly required by the binding of nuclear proteins. In addition, PvERF15 and PvMTF-1 were strongly induced by ethylene in bean seedlings. Since PvERF15 activates PvMTF-1 via ACE, ACE is involved in ethylene-induced PvMTF-1 expression. Taken together, our findings provide genetic and biochemical evidence for a new ERE.
\end{abstract}

Keywords: AC-rich element; ethylene-responsive element; ethylene response factor; Arabidopsis

\section{Introduction}

Gas hormone ethylene regulates numerous development processes and stress responses in plants [1]. The control of these processes by ethylene involves the complex regulation of ethylene biosynthesis, ethylene perception, and signal transduction. A conserved ethylene signaling pathway has been established in model plants such as Arabidopsis (Arabidopsis thaliana) and rice (Oryza sativa) [2]. In the ethylene-induced transcription cascade, the ethylene response factor (ERF) is a crucial component that binds to the GCC box in the promoters of target genes to activate the ethylene response [3]. The GCC box (5'-AGCCGCC-3') was originally identified in the tobacco (Nicotiana tabacum) $\beta$-1,3-glucanase Gln2 gene and is thought to be a common ethylene-responsive element (ERE) [4]. The GCC box is widely contained in the promoter regions of ethylene-inducible defense genes, but not in the regulatory regions of fruit-ripening genes and flower petal senescence genes [5]. Ethylene-responsive promoter regions without the GCC box have been identified in the senescence-related carnation glutathione-S-transferase gene [6] and fruit-ripening tomato E4 gene [7], indicating that the GCC box may not be the only ERF-binding element in the process of ethylene-regulated transcription.

Our previous work described a 19-bp AC-rich element (ACE, 5'-CCTAAACCCCAAAACAATC-3') in the promoter of bean (Phaseolus vulgaris) metal response element-binding transcription factor 1 (PvMTF-1) [8]. The bean ethylene response factor 15 (PvERF15) directly binds ACE to activate PvMTF-1 expression [8]. Interestingly, this newly identified ERF-binding element, ACE, exhibits different properties from the GCC box. Thus, it is of great interest to further investigate whether 
ACE acts as an ERE. In this study, we characterized ACE as a new functional ERE in Arabidopsis. Our findings extend our knowledge of ethylene-regulated transcription.

\section{Results}

\subsection{ACE Causes the Minimal Promoter to Respond to Ethylene in Arabidopsis Seedlings}

To check whether ACE acts as an ERE in plants, we performed the transient $\beta$-glucuronidase (GUS) expression assay in the etiolated Arabidopsis seedlings. Ethylene stress was achieved by applying the ethylene precursor 1-aminocyclopropane-1-carboxylic acid (ACC) [9], whereas endogenous ethylene perception was blocked with $\mathrm{Ag}^{+}$(an inhibitor of the ethylene receptors) [10]. Results showed that $10 \mu \mathrm{M}$ ACC treatment produced a typical ethylene-responsive phenotype. It significantly reduced the hypocotyl or root length of the etiolated Arabidopsis seedlings (Figure 1A). However, the $\mathrm{Ag}^{+}$(from $50 \mu \mathrm{M} \mathrm{AgNO}$ )-treated etiolated Arabidopsis seedlings significantly increased in hypocotyl length due to the inhibition of endogenous ethylene action (Figure 1A). These observations are consistent with previous studies [11].

Next, these seedlings were transfected with the GUS reporter construct (Figure 1B), in which GUS was driven by two copies of ACE or mutated ACE (5'-CCTAAACCCCAAAACAATC- $3^{\prime}$ mutated to $5^{\prime}$-ttTAAAttttAAAAtAATt-3' , named mACE [8]) fused at the upstream of the minimal cauliflower mosaic virus $35 S$ promoter $\left(35 S_{(-46 /+8)}\right)$. A $35 S$ : GFP construct was used as an internal control by co-transformation to normalize the transformation efficiency in each experiment. We found that for Arabidopsis seedlings transfected with ACE-35S $(-46 /+8)$ :GUS, the GUS activity was induced by approximately 2-fold in the presence of ACC, while $\mathrm{AgNO}_{3}$ treatment decreased the GUS activity by about $40 \%$ (Figure 1C). A significant change in GUS activity was not observed in the plants transfected with $m A C E-35 S_{(-46 / 8)}$ :GUS (Figure 1C), suggesting that ACE lost its ethylene responsiveness due to the mutation. These results showed that ACE allows $35 S_{(-46 /+8)}$ to respond to both the ethylene stress and inhibition of ethylene in the etiolated Arabidopsis seedlings. We also noted that plants expressing $A C E-35 S_{(-46 /+8)}$ :GUS exhibited higher GUS accumulation than plants expressing $m A C E-35 S_{(-46 /+8)}$ :GUS (Figure 1C, control lanes). This observation is consistent with previous studies that demonstrate that ACE acts as a positive element in transient GUS assays [8].

\subsection{ACE Specifically Binds to the Nuclear Proteins of Arabidopsis}

Next, we performed electrophoretic mobility shift assay (EMSA) to determine whether ACE interacts specifically with the etiolated Arabidopsis nuclear proteins. The nuclear proteins were immunologically verified (Figure 2A). As a positive and negative control, ACE bound to glutathione S-transferase (GST)-PvERF15 but not the GST mock [9]. We observed that ACE is able to be bound by nuclear proteins (Figure 2B). The ACE-protein complex was significantly inhibited by the addition of a 50 -fold molar excess of unlabeled $2 \times$ ACE (Figure 3A, lanes 1 and 8). We therefore conclude that ACE specifically binds to the nuclear proteins in vitro.

\subsection{The First 9-bp Fragment of ACE Is Required by Nuclear Protein Binding}

To further identify the specific sequence of ACE for nuclear protein binding, a competitive EMSA assay was employed. A series of three-base sequential substitution ACE versions (ACE-M1 to -M6) was used as the competitors (Figure 3A). ACE-M2, -M4, -M5, and -M6 were able to completely inhibit the interaction of nuclear proteins with the ACE probe. However, ACE-M1 and ACE-M3 showed a relatively weaker inhibitory effect on nuclear protein binding. Thus, the 1-3 and 6-9 base pairs of ACE appear essential for nuclear protein binding. We named this first 9-bp sequence (5'-CCTRRRCCC- $3^{\prime}$, $\mathrm{R}=\mathrm{A} / \mathrm{G})$ as $\mathrm{ACE}_{\text {core }}$. 

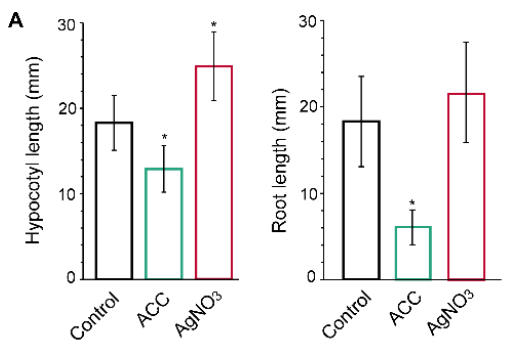

B

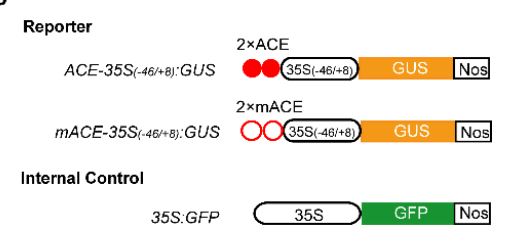

C

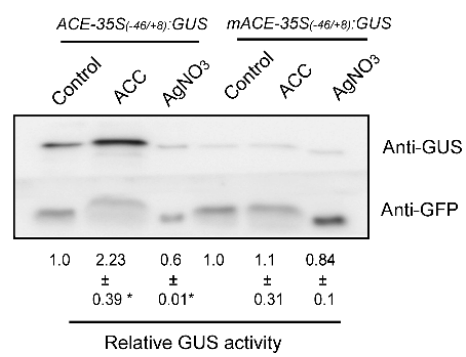

Figure 1. GUS transient assays for ACE as an ERE in the etiolated Arabidopsis seedlings. (A) The Arabidopsis seeds were grown in $1 / 2$ glutathione S-transferase (MS) without (control) and with $10 \mu \mathrm{M}$ $\mathrm{ACC}$ (ACC) or $50 \mu \mathrm{M} \mathrm{AgNO} 3\left(\mathrm{AgNO}_{3}\right)$ in the dark for two weeks and then subjected to hypocotyl or root length analysis. Data are means \pm standard error $(\mathrm{SE})(n=100)$. The significance was assessed using a one-sided Student's $t$-test $\left({ }^{*} p \leq 0.05\right)$. (B,C) GUS transient assays in the etiolated Arabidopsis seedlings. Schematic diagram of constructs used in the experiments (B). The etiolated Arabidopsis seedlings were co-transformed with reporter and internal control constructs. The transformed etiolated Arabidopsis seedlings were grown on the solution medium without (control) or with $10 \mu \mathrm{M} \mathrm{ACC} \mathrm{(ACC)}$ or $50 \mu \mathrm{M} \mathrm{AgNO}_{3}\left(\mathrm{AgNO}_{3}\right)$ for 2 days. The expression of GUS or GFP was determined by immunoblot using an anti-GUS antibody and anti-GFP antibody from the same protein samples. (C) Relative GUS activity was measured as the relative band intensity of GUS to GFP (the control was set as 1). The data represent an average from two independent experiments. Means \pm SE are shown. The significance was assessed by a one-sided Students $t$-test $\left.{ }^{*} p \leq 0.05\right)$.

A

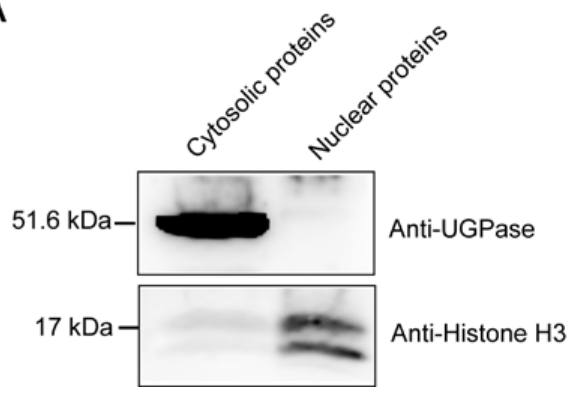

B

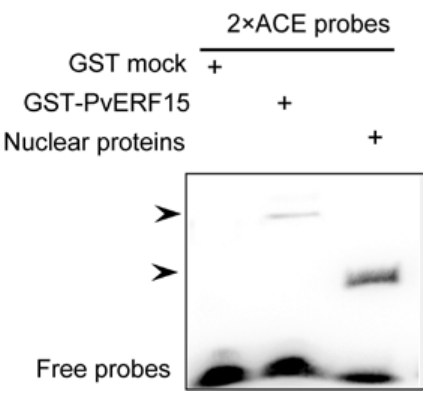

Figure 2. ACE specifically binds to the nuclear proteins in vitro. (A) Western blot verified the successful isolation of nuclear proteins using an anti-histone H3 (nuclei marker) antibody and anti-glucose pyrophosphorylase (UGPase) (cytoplasm marker) antibody. (B) EMSA was performed using biotin-labeled $2 \times$ ACE probes with the affinity-purified GST-PvERF15 (positive control), GST mock (negative control), and nuclear proteins from the etiolated Arabidopsis seedlings. The bound complex is indicated by the arrows. The experiments were performed two times showing similar results. 
A

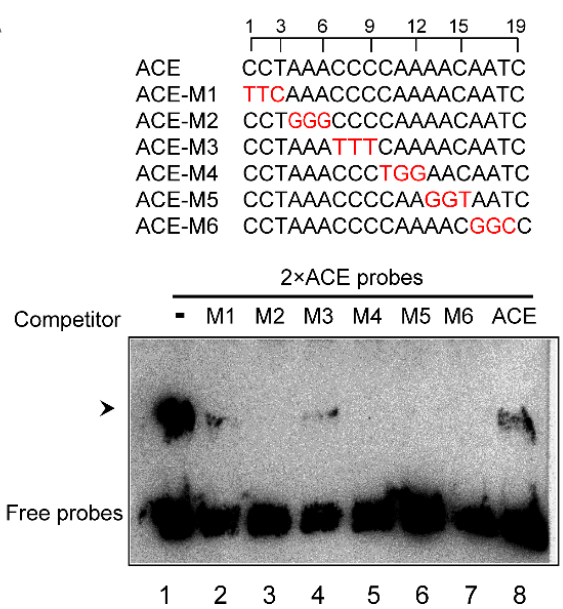

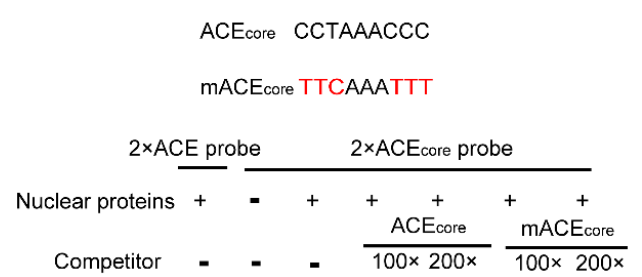

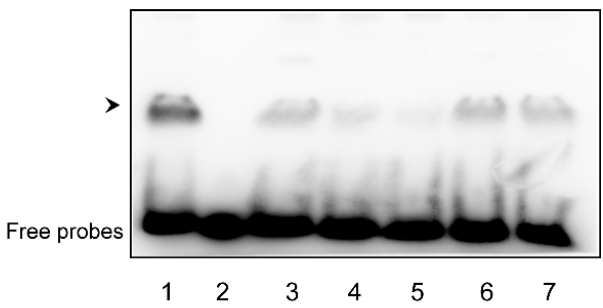

Figure 3. Competitive EMSA identifies the required sequences of ACE for nuclear protein binding. (A) ACE $(2 \times)$ was used as the probe in the EMSA with the nuclear proteins, and the mutant variants (red cases) were used as competitors (50-fold molar). (B) Gel shifts with the $\mathrm{ACE}_{\text {core }}$ probe (2X) showing specific binding to the same and/or similar nuclear proteins as ACE. Mutations introduced into the $A C E_{\text {core }}$ are shown in red. A repeat experiment confirmed the results (Figure $\mathrm{S} 1$ ).

Since $\mathrm{ACE}_{\text {core }}$ produced a similar shifted band to that produced by $\mathrm{ACE}$ (Figure 3B, lanes 1 and 3), $A C E_{\text {core }}$ and $\mathrm{ACE}$ seem to interact with the same and/or similar DNA-binding proteins. An excessive amount of $\mathrm{ACE}_{\text {core }}$ (Figure 3B, lanes 4 and 5), but not mutated $\mathrm{ACE}_{\text {core }}\left(\mathrm{mACE} \mathrm{E}_{\text {core, }}\right.$, Figure 3B, lanes

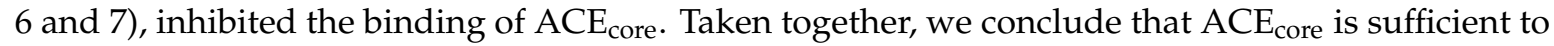
specifically bind with nuclear proteins.

\subsection{PvMTF-1 and PvERF15 Respond to Ethylene Stress in Bean Seedlings}

Since ACE serves as an ERE in Arabidopsis, we investigated whether ACE-containing PvMTF-1 and its transcription regulator PvERF15 are regulated by ethylene in the etiolated bean seedlings (Figure 4A). Treatment with ACC significantly increased the mRNA levels of both PvMTF-1 and PvERF15 (Figure 4B), suggesting that PvMTF-1 and PvERF15 responded to ethylene stress. Given that PvERF15 is located the upstream of PvMTF-1 and acts as a transcription regulator of PvMTF-1 via its ACE [8], ACE is involved in ethylene-induced PvMTF-1 expression.

A

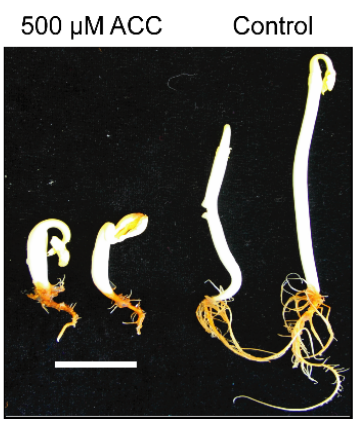

B

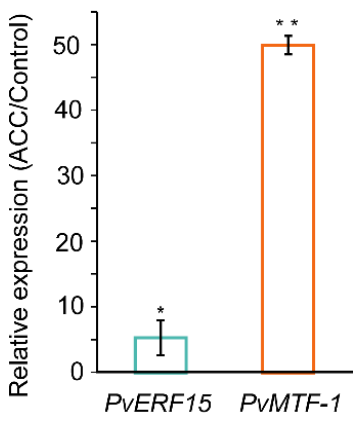

Figure 4. ACC regulates the mRNA levels of both PvMTF-1 and PvERF15 in bean seedlings. (A) Bean seeds were germinated and grown on the MS without (control) or with $500 \mu \mathrm{M}$ ACC for 10 days in the dark. ACC induced a typical ethylene-responsive phenotype, indicating the effects of ethylene stress. Bar $=1 \mathrm{~cm}$. (B) The etiolated bean seedlings were subjected to quantitative reverse transcription qRT-PCR analysis. ACC-induced expression level of PvERF15 or PvMTF-1 was expressed as a ratio relative to control, which was set to a value of 1 . Data shown are averages of three independent qRT-PCR experiments. Error bars represent SEs. Significance between experimental values was assessed using one-sided Student's $t$ test $\left({ }^{*}, p \leq 0.05\right.$, and $\left.{ }^{* *}, p \leq 0.01\right)$. 


\section{Discussion}

\subsection{Our Findings Provide Evidence for ACE as a Novel ERE in Arabidopsis}

In this study, we provided evidence supporting ACE as an ERE in Arabidopsis seedlings. In the transient GUS assay, ACE was able to confer ethylene stress responsiveness on a minimal promoter, whereas the mutated ACE was not (Figure 1C). When the endogenous ethylene perception was blocked by $\mathrm{Ag}^{+}$, ACE-mediated GUS expression was also inhibited (Figure 1C). This reflects the effects of ethylene-induced inhibition of ACE-mediated GUS expression. These findings suggested that ACE-mediated GUS expression is regulated by ethylene stress and ethylene action and, thus, ACE acts as an ERE in Arabidopsis. This can further be supported by the observation that ACE serves as a cis-acting element to specifically bind with nuclear proteins (Figure 2B). In this study, genetic evidence for ACE as an ERE is mainly based on GUS transient assay. To obtain a more complete understanding of ACE function in ethylene response, stably transformed Arabidopsis lines will be needed for future studies.

Given that the $\mathrm{ACE}_{\mathrm{core}}$ sufficiently supports the $\mathrm{ACE}$ binding with the nuclear proteins (Figure $3 \mathrm{~A}, \mathrm{~B}), \mathrm{ACE}_{\mathrm{core}}$, to some extent, may represent $\mathrm{ACE}$. To assess the $\mathrm{ACE}_{\mathrm{core}}$ distribution in the promoter regions $(-1000 \mathrm{bp})$ of Arabidopsis genes, a genome-wide survey was performed. We found that 847 Arabidopsis genes contain $1007 \mathrm{ACE}_{\mathrm{core}}$ in their promoters (Table S1). ACE $\mathrm{E}_{\mathrm{core}}$ appears to be able to globally regulate gene expression in Arabidopsis. However, gene ontology (GO) term enrichment analysis found no significant enrichment for $847 \mathrm{ACE}_{\mathrm{core}}$-containing genes. In the future, further assessment of the regulatory role of $\mathrm{ACE}_{\mathrm{core}}$ in these genes could elucidate the biological significance of ACE.

\subsection{The Biological Implication of ACE in the Ethylene Induction of PvMTF-1}

Our previous study demonstrated that ACE acts as a positive element through which PvERF15 activates PvMTF-1 expression [8]. In this study, we showed that ethylene stress transcriptionally induced the expression of PvERF15 and PvMTF-1 in bean seedlings (Figure 4B). Additionally, other known ERF-binding sites [12] were not found in the 397-bp PvMTF-1 promoter [8,13], implying that ACE seems to be a unique ERE for PvMTF-1. Given this, we propose that upon ethylene stress, PvERF15 expression is induced and then enhances PvMTF-1 expression via directly binding on ACE. Although we cannot exclude the other transcription regulators of PvMTF-1 ethylene-induced expression, ACE interaction with PvERF15 may contribute to the ethylene induction of PvMTF-1, and thus demonstrate the biological implication of ACE in the ethylene induction of PvMTF-1.

In conclusion, the GCC box and ACE share the features of ERF binding and ethylene response. However, in contrast to the high ethylene-inducible ability of the GCC box [4], ACE has a low induction ability (about two-fold induction). Despite this, our findings provide evidence for a new ERE and extend our knowledge of ethylene-regulated transcription.

\section{Materials and Methods}

\subsection{Plant Materials and Growing Conditions}

Seeds of Arabidopsis thaliana (Columbia-0) or bean (Phaseolus vulgaris) were surface-sterilized. Arabidopsis seeds were grown on 1/2 MS agar plates without (as a control) or with $10 \mu \mathrm{M}$ ACC or $50 \mu \mathrm{M}$ $\mathrm{AgNO}_{3}$ in the dark (wrapped in aluminum foil) at $22{ }^{\circ} \mathrm{C}$ for $14 \mathrm{~d}$. The etiolated Arabidopsis seedlings were subjected to hypocotyl length analysis, root length analysis, transient expression assay, or nuclear protein extraction. Bean seeds were grown on MS agar plates without (as a control) or with $500 \mu \mathrm{M}$ ACC in the dark at $22^{\circ} \mathrm{C}$ for $10 \mathrm{~d}$. The etiolated bean seedlings were subjected to RNA isolation. 


\subsection{Agrobacterium Tumefaciens-Mediated Gene Transfer by Infiltration of Arabidopsis Seedlings}

ACE-35S $(-46 /+8)$ :GUS, ACEm-35S $(-46 /+8)$ :GUS, or 35S:GFP (pCAMBIA1302) was used for the transient expression assay as described previously [8]. The etiolated Arabidopsis seedlings were vacuum-infiltrated with $A$. tumefaciens as described previously by Marion et al. [14]. Briefly, A. tumefaciens cells were collected and resuspended at an optical density (OD $600 \mathrm{~nm}=2.0$ ) in $1 / 4$ MS liquid medium containing $5 \%(w / v)$ sucrose, $200 \mathrm{mM}$ acetosyringone, and $0.01 \%(v / v)$ Silwet. After vacuum infiltration, transformed Arabidopsis seedlings were transferred to filter paper soaked with $1 / 4$ MS solution medium with or without $10 \mu \mathrm{M}$ ACC or $50 \mu \mathrm{MAgNO}$ in the dark (wrapped with aluminum foil) at $22^{\circ} \mathrm{C}$ for $2 \mathrm{~d}$.

\subsection{Protein Extraction}

Total protein from the plant samples was extracted using extraction buffer $(50 \mathrm{mM}$ Tris- $\mathrm{HCl})$ pH 7.5), $150 \mathrm{mM} \mathrm{NaCl}, 1 \mathrm{mM}$ EDTA, $10 \%(v / v)$ glycerol, $5 \mathrm{mM}$ dithiothreitol, $0.5 \%(v / v)$ Triton X-100, $1 \mathrm{mM}$ phenylmethylsulphonyl fluoride, and $1 \%(v / v)$ Nonidet P-40).

Nuclear fractionation was performed based on the protocol described by Xia et al. [15] with some modifications. Briefly, $2 \mathrm{~g}$ of etiolated Arabidopsis seedlings was homogenized in Honda buffer (2.5\% Ficoll 400, 5\% dextran T40, $0.4 \mathrm{M}$ sucrose, $25 \mathrm{mM}$ Tris- $\mathrm{HCl}, \mathrm{pH} 7.4,10 \mathrm{mM} \mathrm{MgCl}, 10 \mathrm{mM}$ $\beta$-mercaptoethanol, and a proteinase inhibitor cocktail) using a mortar and pestle. The homogenate was filtered through two layers of $64-\mu \mathrm{m}$ (pore-size) nylon mesh. Triton X-100 was added to a final concentration of $0.5 \%$, and the mixture was incubated on ice for $15 \mathrm{~min}$. The mixture was centrifuged at $1500 \times \mathrm{g}$ for $5 \mathrm{~min}$. The supernatants were used as the cytosolic protein, and the pellets were washed twice in Honda buffer containing $0.1 \%$ Triton X-100 and used as the nuclei-enriched fraction. This nucleus-enriched preparation was centrifuged at $100 \times g$ for $5 \mathrm{~min}$ to pellet the starch and cell debris. The supernatant was centrifuged subsequently at $2000 \times g$ for $5 \mathrm{~min}$ to pellet the nuclei. The purified nuclei were resuspended gently in $125 \mu \mathrm{L}$ of Honda buffer and transferred to a microcentrifuge tube.

GST-PvERF15 fusion protein or GST protein (mock) was prepared as previously described [8].

\subsection{EMSA}

The $5^{\prime}$ biotin-labeled DNA probes or the corresponding unlabeled DNA were synthesized by Sangon Biotechnology (Shanghai, China). The top strand and the bottom strand were responded in the annealing buffer (50 mM Tris- $\mathrm{HCl}(\mathrm{pH} 7.5), 250 \mathrm{mM} \mathrm{NaCl}, 0.5 \mathrm{mM}$ EDTA) at a 1:1 ratio. Heat the mixture to $95^{\circ} \mathrm{C}$ for $4 \mathrm{~min}$ to remove all secondary structures, and then anneal it at $60-70{ }^{\circ} \mathrm{C}$ for $20 \mathrm{~min}$. Following this, gradually decrease (such as $5^{\circ} \mathrm{C} / \mathrm{min}$ ) the temperature to $25^{\circ} \mathrm{C}$ and maintain this for 1-2 h. EMSA was performed using the LightShift Chemiluminescent EMSA Kit (Pierce) according to the manufacturer's instructions. A total of $100 \mathrm{ng}$ of nuclear proteins was incubated together with $1.5 \mathrm{ng}$ of biotin-labeled probes in $25-\mu \mathrm{L}$ reaction mixtures $(1 \times$ binding buffer, $50 \mathrm{ng}$ poly(deoxyinosinic-deoxycytidylic acid), 2.5\% $(v / v)$ glycerol, $0.05 \%(v / v)$ Nonidet P-40, and $5 \mathrm{mM}$ $\mathrm{MgCl}_{2}$ ) for $30 \mathrm{~min}$ at room temperature. For the cold competitor, the unlabeled competitors were added into the reaction mixture. The reaction mixtures were separated on $6 \%(w / v)$ native PAGE gels. The labeled probes were detected according to the instructions provided with the EMSA kit using a Fujifilm LAS-3000 imager.

\subsection{Western Blot Analysis}

Proteins separated on a gel were electrophoretically transferred to a pure nitrocellulose blotting membrane (Pall Life Sciences). The membrane was cut across the molecular mass region of the corresponding proteins and separately probed with an anti-GUS antibody (ab50148, Abcam), an anti-GFP antibody (ab290, Abcam), or anti-tubulin antibody (T5168, Sigma-Aldrich), and an anti-histone H3 antibody (AS10 710, Agrisera) or an anti-UGPase antibody (AS05 086, Agrisera). Protein blots were developed with an ECL kit (Amersham Pharmacia Biotech), and images were 
obtained using the LAS3000 image-capture system (Fujifilm). The Adobe Photoshop CS histogram analysis tool was used for the grayscale analysis of the Western blot bands of GUS and GFP.

\subsection{RNA Isolation and cDNA Synthesis}

Total RNA was extracted from the etiolated bean seedlings using the RNAprep pure plant kit with on-column DNase digestion (Tiangen Biotech, China). RNA (about $2 \mu \mathrm{g}$ ) was used to synthesize the first-strand cDNA with oligo(dT) primers with the PrimeScript first-strand cDNA synthesis kit (Takara).

\subsection{Real-Time Quantitative Reverse Transcription ( $q R T)-P C R$ Analysis}

The qRT-PCR analysis of PvERF15 (accession number: XM_007144842) or PvMTF-1 (accession numbers: DQ109993 and U54704) was performed on a the CFX96 Real-Time System (Bio-Rad) with the SYBR Premix Ex-Taq Kit (Takara) according to the manufacturer's instructions. The bean ACTIN gene (accession number: AB067722) was used as an internal control. The thermal cycling conditions were $95{ }^{\circ} \mathrm{C}$ for $3 \mathrm{~min}$ followed by 50 cycles of $95{ }^{\circ} \mathrm{C}$ for $30 \mathrm{~s}, 55{ }^{\circ} \mathrm{C}$ for $30 \mathrm{~s}$, and $72{ }^{\circ} \mathrm{C}$ for $30 \mathrm{~s}$. The primers used for qRT-PCR were as follows: PvERF15-F (5'TGGCACTCAAGAGGAAACACAC-3') and PvERF15-R (5'-CATTCTCCAACTGGTGCTCCC-3') for PvERF15, PvMTF-1-F (5'-CTGAAGAGTTGCGAATTGCACGAA-3') and PvMTF-1-R (5'-TTGAATTTGATTGAATCTTGCAGGATG-3') for PvMTF-1, and Actin-F (5'CACCGAGGCACCGCTTAATC-3') and Actin-R (5'-CGGCCACTAGCGTAAAGGGAA-3') for ACTIN. The relative expression levels were analyzed using a delta-delta cycle threshold method.

\subsection{Bioinformatics Analysis}

Using CCTRRRCCC ( $\mathrm{R}=\mathrm{A}$ or $\mathrm{G})$ as a query, the Patmatch program available at the TAIR web site (http://www.arabidopsis.org/cgi-bin/patmatch/nph-patmatch.pl) was employed to search

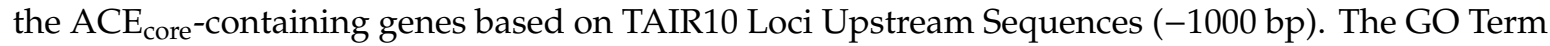
Enrichment for Plants tool (https://www.arabidopsis.org/tools/go_term_enrichment.jsp) was used for $847 \mathrm{ACE}_{\text {core-containing genes. }}$

\subsection{Statistical Analysis}

Statistical analysis (SE and $p$-values) was conducted using Microsoft Excel 2007. Significance $(p<0.05)$ was assessed using Student's t-tests.

Supplementary Materials: The following are available online at http://www.mdpi.com/2223-7747/9/8/1033/s1, Figure S1. The repeat EMSAs confirming the results in Figure 3. Table S1: A total of 847 genes contain ACEcore in their promoter regions (-1000 bp) in Arabidopsis.

Author Contributions: X.Q. conceived the research; T.L., M.W. and C.W. performed the research and analyzed the data; and C.W. and X.Q. wrote the paper. All authors have read and agreed to the published version of the manuscript.

Funding: This research was funded by the National Natural Science Foundation of China (grant nos. 31771361 and 31271293 to X.Q.) and the general science and the Scientific Research Program of Beijing Education Commission (grant no. SQKM201810028011 to X.Q.).

Acknowledgments: We thank LetPub for its linguistic assistance during the preparation of this manuscript. We thank Weiwei Zhang (Capital Normal University, China) for critical reading and valuable suggestions. We also thank Yan Li and Xudong Shang (Capital Normal University, China) for technical assistance.

Conflicts of Interest: The authors declare no conflict of interest. 


\section{References}

1. Dubois, M.; Van den Broeck, L.; Inzé, D. The pivotal role of ethylene in plant growth. Trends Plant. Sci. 2018, 23, 311-323. [CrossRef] [PubMed]

2. Yang, C.; Lu, X.; Ma, B.; Chen, S.Y.; Zhang, J.S. Ethylene signaling in rice and Arabidopsis: Conserved and diverged aspects. Mol. Plant 2015, 8, 495-505. [CrossRef] [PubMed]

3. Solano, R.; Stepanova, A.; Chao, Q.; Ecker, J.R. Nuclear events in ethylene signaling: A transcriptional cascade mediated by ETHYLENE-INSENSITIVE3 and ETHYLENE-RESPONSE-FACTOR1. Genes. Dev. 1998, 12, 3703-3714. [CrossRef] [PubMed]

4. Ohme-Takagi, M.; Shinshi, H. Ethylene-inducible DNA binding proteins that interact with an ethyleneresponsive element. Plant Cell 1995, 7, 173-182. [PubMed]

5. Ohme-Takagi, M.; Suzuki, K.; Shinshi, H. Regulation of ethylene-induced transcription of defense genes. Plant Cell Physiol. 2000, 41, 1187-1192. [CrossRef] [PubMed]

6. Itzhaki, H.; Maxson, J.M.; Woodson, W.R. An ethylene-responsive enhancer element is involved in the senescence-related expression of the carnation glutathione-S-transferase (GST1) gene. Proc. Natl. Acad. Sci. USA 1994, 91, 8925-8929. [CrossRef] [PubMed]

7. Montgomery, J.; Goldman, S.; Deikman, J.; Margossian, L.; Fischer, R.L. Identification of an ethylene-responsive region in the promoter of a fruit ripening gene. Proc. Natl. Acad. Sci. USA 1993, 90, 5939-5943. [CrossRef] [PubMed]

8. Lin, T.; Yang, W.; Lu, W.; Wang, Y.; Qi, X. Transcription factors PvERF15 and PvMTF-1 form a cadmium stress transcriptional pathway. Plant Physiol. 2017, 173, 1565-1573. [CrossRef] [PubMed]

9. Peiser, G.D.; Wang, T.T.; Hoffman, N.E.; Yang, S.F.; Liu, H.W.; Walsh, C.T. Formation of cyanide from carbon 1 of 1-aminocyclopropane-1-carboxylic acid during its conversion to ethylene. Proc. Natl. Acad. Sci. USA 1984, 81, 3059-3063. [CrossRef] [PubMed]

10. Beyer, E.M. Effect of silver, carbon dioxide, and oxygen on ethylene action and metabolism. Plant Physiol. 1979, 63, 169-173. [CrossRef] [PubMed]

11. Cary, A.J.; Liu, W.; Howell, S.H. Cytokinin action is coupled to ethylene in its effects on the inhibition of root and hypocotyl elongation in Arabidopsis thaliana seedlings. Plant Physiol. 1995, 107, 1075-1082. [CrossRef] [PubMed]

12. Sasaki, K.; Mitsuhara, I.; Seo, S.; Ito, H.; Matsui, H.; Ohashi, Y. Two novel AP2/ERF domain proteins interact with cis-element VWRE for wound-induced expression of the tobacco tpoxN1 gene. Plant J. 2007, 50, 1079-1092. [CrossRef] [PubMed]

13. Qi, X.T.; Zhang, Y.X.; Chai, T.Y. The bean PvSR2 gene produces two transcripts by alternative promoter usage. Biochem. Biophys. Res. Commun. 2007, 356, 273-278. [CrossRef] [PubMed]

14. Marion, J.; Bach, L.; Bellec, Y.; Meyer, C.; Gissot, L.; Faure, J.D. Systematic analysis of protein subcellular localization and interaction using high-throughput transient transformation of Arabidopsis seedlings. Plant J. 2008, 56, 169-179. [CrossRef] [PubMed]

15. Xia, Y.; Nikolau, B.J.; Schnable, P.S. Developmental and hormonal regulation of the Arabidopsis CER2 gene that codes for a nuclear-localized protein required for the normal accumulation of cuticular waxes. Plant Physiol. 1997, 115, 925-937. [CrossRef] [PubMed]

(C) 2020 by the authors. Licensee MDPI, Basel, Switzerland. This article is an open access article distributed under the terms and conditions of the Creative Commons Attribution (CC BY) license (http://creativecommons.org/licenses/by/4.0/). 